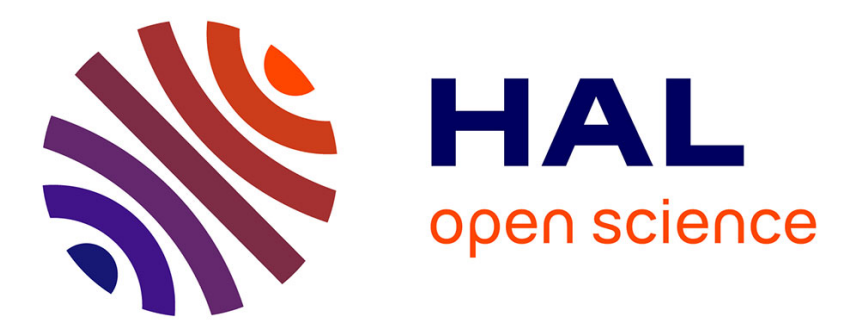

\title{
Fault-Tolerant Flight Control Based on Two-Stage Approach and Interior Point Method
}

Lunlong Zhong, Alexandre Carlos Brandao-Ramos, Felix Mora-Camino

\section{To cite this version:}

Lunlong Zhong, Alexandre Carlos Brandao-Ramos, Felix Mora-Camino. Fault-Tolerant Flight Control Based on Two-Stage Approach and Interior Point Method. Wireless Personal Communications, 2018, 102 (2), pp 1809-1825. 10.1007/s11277-017-5238-4 . hal-02010869

\section{HAL Id: hal-02010869 \\ https://hal-enac.archives-ouvertes.fr/hal-02010869}

Submitted on 7 Feb 2019

HAL is a multi-disciplinary open access archive for the deposit and dissemination of scientific research documents, whether they are published or not. The documents may come from teaching and research institutions in France or abroad, or from public or private research centers.
L'archive ouverte pluridisciplinaire HAL, est destinée au dépôt et à la diffusion de documents scientifiques de niveau recherche, publiés ou non, émanant des établissements d'enseignement et de recherche français ou étrangers, des laboratoires publics ou privés. 


\title{
Aircraft Fault Tolerant Control Based on Two-stage Approach and Interior Point Method
}

\author{
Lunlong Zhong ${ }^{1}$, Alexandre Carlos Brandão Ramos ${ }^{2}$, Félix Mora-Camino ${ }^{3}$ \\ 1. Tianjin Key Laboratory for Advanced Signal Processing, Civil Aviation University of China, Tianjin 300300, China \\ E-mail: zlunlong@163.com
}

2. Mathematical and Computer Science Institute, Federal University of Itajuha, 37500-900, Brazil.

E-mail: $\underline{\text { ramos@unifei.edu.br }}$

3. ENAC, Toulouse University, 7 avenue Edouard Belin, 31055 Toulouse, France

E-mail: felix.mora@enac.fr

\begin{abstract}
The main objective of this paper is how to make the best use of remaining available actuators when an aircraft with an aerodynamic actuator failure tries to perform a guidance maneuver. Considering the flight dynamics of a transportation aircraft can be expressed as an input affine nonlinear system, a two-stage control approach is adopted here. At the first stage, nonlinear inverse control technique is used to generate virtual inputs related to the intended maneuver. At the second stage, an actuator reassignment problem how to achieve necessary virtual inputs by remaining available actuators is considered. Taking into account the physical limits of the remaining actuators and of the aircraft structure, the problem is formulated as a quadratic programming problem to get the corresponding control signals sent to the remaining actuators. The proposed solution method for this quadratic mathematical programming problem is based on a primal-dual path following interior point method. Numerical simulation studies based on the nonlinear aircraft models show that the proposed solution approach appears to provide acceptable response times to perform accurately the guidance maneuver.
\end{abstract}

Key Words: Fault tolerant control, Flight control, Two-stage approach, Interior point method, Actuator reassignment

\section{Introduction}

In this paper, we consider the situation in which a main aerodynamic actuator of a transportation aircraft fails while it has to perform a guidance maneuver. To make the best use of the remaining operational actuators, a two-stage control approach [1] is adopted. Using dynamic inversion of flight dynamics, the virtual inputs necessary to perform a given guidance maneuver are computed, and then an optimization problem is considered to generate on-line reference values for the remaining actuators. This represents the main difference with other previous approaches to actuator fault management [2-4]. In the case considered here, an on-line linear quadratic programming formulation of the optimization problem can be adopted and an interior point approach to get an on-line solution is discussed. The approach is also different from the one we proposed in [5] which is based on the use of a neural network dynamic solver.

\section{A Two-stage Control Approach}

In this paper, a two-stage control approach proposed in [1] is adopted. The following is a simple introduction of this two-stage control approach. For the detail, see ref. [1].

Many nonlinear dynamical systems such as aircraft can be modeling as an input affine form:

This work is supported by Tianjin Research Program of Application Foundation and Advanced Technology for Youths (Grant No. 15JCQNJC04400), Fundamental Research Funds for the Central Universities (Grant No. 3122015C004)

$$
\begin{gathered}
\underline{\&}=f(\underline{x})+\sum_{j=1}^{m} g_{j}(\underline{x}) \cdot u_{j} \quad \underline{x} \in R^{n}, \underline{u} \in R^{m} \\
\underline{y}=h(\underline{x}) \quad \underline{y} \in R^{p}
\end{gathered}
$$

where $\underline{x}$ is the state vector representing the system dynamics, $\underline{u}$ is the control inputs vector, $\underline{y}$ is the chosen independent outputs, $f(\underline{x})$ and $g_{i}(\underline{x}), i=1$ to $m$, are smooth vector fields of $\underline{x}$. $h(\underline{x})$ is a smooth vector field of $\underline{x}$. It is supposed that $p<n$ and $p<m$.

Normally, the desired maneuver for the dynamical systems can be expressed analytical by standard differential equations of desired outputs $\underline{y}$. So we hope the actual outputs can follow the desired trajectory. When the output variables are chosen so that there are no internal dynamics left, the system will be governable. Then, these systems can be fault tolerant trajectory following by the two-stage control approach illustrated by Fig. 1 .

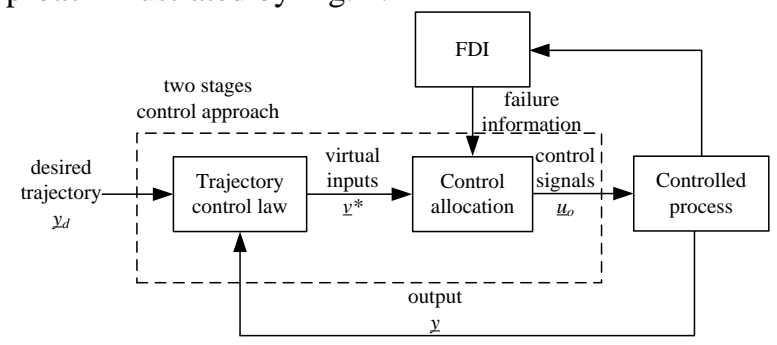

Fig.1 Proposed fault tolerant trajectory tracking scheme.

In the two-stage control approach, the control signal synthesis is split into a virtual inputs synthesis problem for system (1) and a control allocation problem to distribute the virtual inputs among the remaining operational inputs. The 
benefits of adopting such strategy can be stated as: When solving the virtual inputs control problem, it is not yet necessary to take into account the physical constraints attached to each operational actuator. This is fortunate since few control techniques are able to take explicitly into account input constraints. Then the actuator constraints as well as other operational limitations can be taken more easily into account when solving the control allocation problem. Moreover, additional constraints can be taken into account in the control allocation problem.

At the first stage, when control law is chosen as nonlinear inverse control, the desired virtual inputs can be written as:

$$
v_{i}^{*}=y_{d i}^{\left(r_{i}+1\right)}-\sum_{k=0}^{r_{i}} c_{i k}\left(y_{i}^{(k)}-y_{d i}^{(k)}\right)-F_{i}(\underline{X}) \quad i=1 \text { to } p
$$

where $y_{d i}, i=1$ to $p$, is the desired output trajectory while the coefficients $c_{i k}$ are chosen so that the dynamics of the tracking error defined by $e_{i}=y_{i}-y_{d i}, i=1$ to $p$, is asymptotically stable and converges towards zero. $r_{i}$ is the relative degree of output $i$. $\underline{X}$ is a vector of $j^{\text {th }}$ order differential of $y_{i}, j=0$ to $r_{i} . F_{i}(\underline{X})$ is a function of vector $\underline{X}$.

In Eq. (3), assume there are some actuators fail, the actual virtual inputs will be generated by the physical control inputs:

$$
\underline{v}=D_{o}(\underline{X}) \cdot \underline{u}_{o}+D_{f}(\underline{X}) \cdot \underline{u}_{f}
$$

here matrix $D_{o} \in R^{p \times m_{o}}$ is the distribution matrix associated to the identified failure case, $\underline{u}_{o} \in R^{m_{o}}$ is the vector of the remaining operational control inputs, $D_{f} \in R^{p \times m_{f}}$ is the control effectiveness matrix related to the failed control inputs, $\underline{u}_{f} \in R^{m_{f}}$ is the vector of the failed control inputs which are no more active and whose values are supposed known.

Then, at the second stage, a control allocation problem can be formed as how to distribute effects of available actuators to generate the desired virtual inputs as well as to satisfy the physical constraints related to actuators or controlled system. The equality satisfied by efforts distribution can be written as:

$$
D_{o}(\underline{X}) \cdot \underline{u}_{o}+D_{f}(\underline{X}) \cdot \underline{u}_{f}=\underline{v}^{*}
$$

\section{Virtual Inputs Generation for Fast Flight Control}

The fast flight dynamics is also an affine system, so the approach discussed above can be applied when some actuators failure.

\subsection{Aircraft rotation dynamics}

The equation for the angular movement of a rigid aircraft in the body reference frame can be expressed as:

$$
\underline{M}=I_{m} \underline{\alpha}+\underline{\omega} \times\left(I_{m} \underline{\omega}\right)
$$

where $\underline{M}=\left(\begin{array}{lll}L & M & N\end{array}\right)^{\prime}, L, M, N$ are respectively the roll, pitch, and yaw aerodynamic torques, $I_{m}$ is the matrix of inertial moments, $\underline{\omega}$ is the inertial rotational velocity written in the body reference $(p, q, r)$ ' where $p$ is the roll rate, $q$ is the pitch rate and $r$ is the yaw rate, $\underline{\alpha}$ is the inertial rotational acceleration in the body-fixed axis system, $x$ is the cross product operator.

The aerodynamic moments along each body axis are given by:

$L=\frac{1}{2} \rho V^{2} S l C_{l}, M=\frac{1}{2} \rho V^{2} S l C_{m}, N=\frac{1}{2} \rho V^{2} S l C_{n}$

where $C_{l}, C_{m}$ and $C_{n}$ are respectively the roll, pitch and yaw dimensionless aerodynamic coefficients. Here $V$ is the airspeed, $\rho$ is the density of air, $S$ and $l$ are respectively reference area and length specific to the considered aircraft.

The dimensionless coefficients of the main axis aerodynamic torques can in general be expressed such as:

$C_{m}=C_{m 0}+C_{m \alpha} \cdot \alpha+C_{m q} \cdot q l / V+C_{m \delta t h s} \cdot \delta_{t h s}+\underline{C}_{m \delta q}{ }^{\prime} \cdot \underline{\delta}_{q}$

$C_{l}=C_{l 0}+C_{l \beta} \cdot \beta+C_{l p} \cdot p l / V+C_{l r} \cdot r l / V+\underline{C}_{l \delta p} \underline{\delta}_{p}+\underline{C}_{l \delta r} \cdot \underline{\delta}_{r}$

$C_{n}=C_{n 0}+C_{n \beta} \cdot \beta+C_{n p} \cdot p l / V+C_{n r} \cdot r l / V+\underline{C}_{n \delta p}{ }^{\prime} \underline{\delta}_{p}+\underline{C}_{n \delta r} \cdot \underline{\delta}_{r}$

where $\alpha$ is the angle of attack, $\beta$ is the side slip angle, $\underline{\delta}_{p}, \underline{\delta}_{q}$, $\delta_{r}$ are respectively the aileron, elevator and rudder deflections while $\delta_{t h s}$ is the deflection of the trimmable horizontal stabilizer, if any.

\subsection{Effectiveness of redundant actuators}

Considering mainly the size of modern transportation aircraft as well as the reliability issue, an over-redundancy for actuators which contribute to the roll, pitch and yaw moments around three main axes, often exists on modern transportation aircraft. The effectiveness of these actuators appears through the contributions of their angular deflections to these moments through dimensionless coefficients as in equations (6a), (6b) and (6c). According to the relationship between aerodynamic derivatives and aerodynamic torques, the expressions of the different aerodynamic torques generated by these control surfaces can be approximated by affine forms with respect to the corresponding deflections of the different aerodynamic actuators, so that equations related to torques and deflections can be expressed as:

$$
\begin{gathered}
L(t)=L^{0}(t)+\sum_{i \in I^{L}} C_{i}^{L}(t) \delta_{i}(t) \\
M(t)=M^{0}(t)+\sum_{i \in I^{M}} C_{i}^{M}(t) \delta_{i}(t) \\
N(t)=N^{0}(t)+\sum_{i \in I^{N}} C_{i}^{N}(t) \delta_{i}(t)
\end{gathered}
$$

with $I^{a}=I^{L} \cup I^{M} \cup I^{N}$, where $I^{L}$ is the set of actuators generating some roll moment, $I^{N}$ is the set of actuators generating some yaw torque, while $I^{M}$ is the set of actuators generating pitch moments. Here the current values $L^{0}(t), M^{0}(t)$ and $N^{0}(t)$ as well as $C_{i}^{L}(t), C_{i}^{M}(t)$ and $C_{i}^{N}(t)$ depend on the airspeed $V$, the flight level and on the values of $\alpha, \beta, p, q$ and $r$. Global aerodynamic torques generated by aircraft aerodynamic actuators can be rewritten in a global affine form as:

$$
\underline{M}=\underline{M}^{0}+C \cdot \underline{\delta} \quad \text { with } \quad \underline{\delta} \in R^{\left|I^{a}\right|} \text { and } C \in R^{3 \times\left|I^{a}\right|}
$$

\subsection{Generation of virtual inputs}

Eq. (5) can be rewritten as:

$$
\underline{\alpha}=-I_{m}^{-1}\left[\underline{\omega} \times\left(I_{m} \underline{\omega}\right)\right]+I_{m}^{-1} \underline{M}
$$


It is clear that the $p, q$ and $r$ outputs have zero relative degree with respect to $L, M$ and $N$ which can be chosen as virtual inputs. Assuming the aircraft perform a pure roll maneuver where three body angular rates follow the first order dynamics such as:

$$
\begin{gathered}
\tau_{p} \text { \&\&+ } p=p_{c} \\
q=0 \\
\tau_{r} \&+r=(g / V) \sin \varphi
\end{gathered}
$$

Then, applying the nonlinear inverse control approach, the necessary online values for each aerodynamic torque can be written as:

$$
\begin{gathered}
M^{*}(t)=\left(I_{x x}-I_{z z}\right) r(t) p(t)+I_{x z}\left(p(t)^{2}-r(t)^{2}\right) \\
{\left[\begin{array}{c}
L^{*}(t) \\
N^{*}(t)
\end{array}\right]=\left[\begin{array}{cc}
I_{x x} & -I_{x z} \\
-I_{x z} & I_{z z}
\end{array}\right]\left[\begin{array}{c}
\left(p_{c}-p(t)\right) / \tau_{p} \\
((g / V(t)) \sin \phi(t)-r(t)) / \tau_{r}
\end{array}\right]}
\end{gathered}
$$

where $I_{x x}, I_{x z}$, and $I_{z z}$, are the main inertial moments of the aircraft. These necessary online values can be obtained through distribute redundant actuators according to (9).

Here is considered some actuators failure, as expressed in Eq. (4), fault tolerance will exist thanks to the existing actuator redundancy, so the next problem is how to reassignment the remaining effective actuators to produce the required virtual inputs.

\section{Linear Quadratic Formulation for Control Allocation Problem}

When some actuators fail, the maneuver will be performed still in a standard way, otherwise, an approximate maneuver will be performed. In order to get a feasible control allocation which avoids too fast or too large solicitations of the actuators which could activate some structural modes of the aircraft, solutions as close as possible to the solution adopted at the previous control period will be privileged. Also, it is admitted that when the standard maneuver can no more be performed, a close maneuver, in fact a slightly degraded maneuver, will be retained as a running solution. So, instead of considering the pure satisfaction of the moment constraints (8a), (8b) and (8c), a quadratic objective function to measure the degree of satisfaction of these constraints is introduced. Finally the following linear quadratic problem is proposed to solve online:

$$
\min _{\underline{\underline{g}}(t)}\left\{\begin{array}{l}
w_{L}\left(\sum_{i \in I_{k}^{L}} X_{i}^{L}(t) \delta_{i}^{\&}(t)-L^{*}(t)+L^{0}(t)\right)^{2} \\
+w_{M}\left(\sum_{i \in I_{k}^{M}} X_{i}^{M}(t) \delta_{i}^{\&}(t)-M^{*}(t)+M^{0}(t)\right)^{2} \\
+w_{N}\left(\sum_{i \in I_{k}^{N}} X_{i}^{N}(t) \delta_{i}^{\mathcal{Q}}(t)-N^{*}(t)+N^{0}(t)\right)^{2}
\end{array}\right\}
$$

where $\underline{\mathcal{E}} \in R^{\left|I_{k}^{a}\right|} \quad\left(I_{k}^{a}=I_{k}^{L} \cup I_{k}^{M} \cup I_{k}^{N}\right)$ denotes the deflection position of actuators after failure $k$, denoted by subscript $k$, happens.

with the following constraints:

$$
\begin{gathered}
A_{b}(t)+\sum_{i \in I_{k}^{\text {wing }}} Y_{b i}(t) \delta_{i}^{\&} q(t) \leq M_{\text {bend }}^{\max } \\
A_{f}(t)+\sum_{i \in I_{k}^{\text {wing }}} Y_{f i}(t) \delta_{i}^{\&}(t) \leq M_{\text {tors }}^{\max } \\
\delta_{i}^{\text {min }} \leq \delta_{i} \delta^{\circ} \leq \delta_{i}^{\max } \quad i \in I_{\bar{F}_{k}}
\end{gathered}
$$

$$
\begin{aligned}
& \delta_{i}^{g} \leq \delta_{i} \delta_{i} \delta_{i}^{\text {rax }} \quad i \in I_{F L_{k}} \\
& \max \left\{\delta_{i}^{\min }, \delta_{i}^{\mathcal{Q}}(t-\Delta t)+\delta_{i}^{\min } \Delta t\right\} \leq \delta_{i}^{\mathscr{Q}}(t) \quad i \in I_{\bar{F}_{k}} \\
& \delta_{i}(t) \leq \min \left\{\delta_{i}^{\max }, \delta_{i}^{\&}(t-\Delta t)+\delta_{i}^{\max } \Delta t\right\} \quad i \in I_{\bar{F}_{k}} \\
& \max \left\{\delta_{i}^{g}, \delta_{i}^{\&}(t-\Delta t)+\delta_{i}^{\text {in }} \Delta t\right\} \leq \delta_{i}^{\delta}(t) \quad i \in I_{F S_{k}} \\
& \delta(q) \leq \min \left\{\delta_{i}, \delta_{i}(t-\Delta t)+\delta_{i}^{a \mathrm{ax}} \Delta t\right\} \quad i \in I_{F S_{k}} \\
& \text { with } \quad \delta_{i_{j}}^{O o}=0 \text { if } i_{j} \in I_{F F_{k}}, j \in\{p, q, r, t h s\} \\
& \text { and } \quad \delta_{i_{j}} / o=\bar{\delta}_{i_{j}} \text { if } i_{j} \in I_{F P_{k}}, j \in\{p, q, r, t h s\}
\end{aligned}
$$

where $I_{k}^{\text {wing }} \subset I_{k}^{a}$ is the set of remaining operational actuators on wing after failure $k$, constraints (14) are related to total wing bending and torsion moments and should be satisfied to assure the structure integrity. $I_{\bar{F}_{k}}$ is the set of fully operational actuators after failure $k, I_{F L_{k}}, I_{F S_{k}}$ are respectively the set of actuators whose angular positions, angular speed are subject to addition limitations after failure $k, I_{F P_{k}}$ is the set of actuators which are stuck at a known angular position after failure $k, I_{F F_{k}}$ is the set of actuators which are not subject to a torque from their servo-control and with a zero deflection after failure $k . w_{L}, w_{M}$ and $w_{N}$ are positive weighting parameters to priority different torques.

To get an accurate and timely solution for the above linear quadratic (LQ) programming problem, different numerical methods exist. Interior point method mainly studied and examined here.

\section{Interior Point Method Applied to Linear Quadratic Optimization Problems}

Interior point methods and active set methods are the most commonly employed approaches for solving general quadratic programming problems. For a review, see [9], [10] The main advantage of interior point methods over active set method is their scalability [11]. We concentrate in the present study on interior point methods.

The idea of interior point methods is to approach the solution of the Karush-Kuhn-Tucker (KKT) equations by successive descent steps. Each descent step is a Newton-like step and is obtained by solving a system of linear equations.

First, the original problem (14-17) can be written as:

$$
\begin{array}{ll}
\min _{\underline{\delta}} f(\underline{\delta})=\frac{1}{2} \underline{\delta}^{T} Q \underline{\delta}+\underline{c}^{T} \underline{\delta} \\
\text { s.t. } & g(\underline{\delta})=A \underline{\delta}-\underline{b} \leq \underline{0} \\
& \underline{\xi^{-}} \leq \underline{\delta} \leq \underline{\xi}^{+}
\end{array}
$$

where $\underline{\delta}, \underline{\xi}^{-}, \underline{\xi}^{+}, \underline{c}$ are $n \times 1$ column vectors, $\underline{b}$ is a $m \times 1$ column vector, $Q$ is a $n \times n$ matrix, and $A$ is a $m \times n$ matrix, superscript $T$ means the transpose. In our case, $n, m$ are the numbers of actuators and structural constraints respectively. To turn problem $(17,18)$ into a standard form, let

$$
\underline{\delta}=\underline{x}+\underline{\xi}
$$

Substituting (19) in (17) and (18), and omitting the constant item in the objective function which will not impact the final result, the original problem is equivalent to the following problem: 


$$
\begin{array}{ll}
\min _{\underline{x}} & f(\underline{x})=\frac{1}{2} \underline{x}^{T} Q \underline{x}+\underline{x}^{T} \underline{\mathscr{g}} \\
\text { s.t. } & g(\underline{x})=A \underline{x}+\underline{b}^{O} \leq \underline{0} \\
& \underline{0} \leq \underline{x} \leq \underline{x}^{+}
\end{array}
$$

where $\underline{g} \sigma=Q \underline{\xi}^{-}+\underline{c}, \underline{b}^{\circ}=A \underline{\xi}^{-}-\underline{b}$ and $\underline{x}^{+}=\underline{\xi}^{+}-\underline{\xi}^{-}$.

Adding slack variables $\underline{y}, \underline{z}$ to turn inequalities into equalities, we get the following formulation:

$$
\begin{array}{ll} 
& \min _{\underline{x}} \frac{1}{2} \underline{x}^{T} Q \underline{x}+\underline{x}^{T} \underline{\theta} \\
\text { s.t. } & A \underline{x}+\underline{b}^{b} \underline{y}=\underline{0}, \quad \underline{x}+\underline{z}=\underline{x}^{+} \\
& \underline{x} \geq \underline{0}, \underline{y} \geq \underline{0}, \underline{z} \geq \underline{0}
\end{array}
$$

One of the basic ideas behind the interior point methods is to use barrier functions to satisfy the bound constraints. Then the modified Lagrangian of problem $(22,23)$ is expressed as:

$$
\begin{aligned}
& L(\underline{x}, \underline{y}, \underline{z})=\frac{1}{2} \underline{x}^{T} Q \underline{x}+\underline{x}^{T} \underline{q} \theta-\tau \sum_{i=1}^{n} \log \left(x_{i}\right)-\tau \sum_{i=1}^{k} \log \left(y_{i}\right) \\
& -\tau \sum_{i=1}^{n} \log \left(z_{i}\right)+\underline{\mu}^{T}\left(A \underline{x}+\underline{b}^{q} \underline{y}\right)+\underline{\varphi}^{T}\left(\underline{x}+\underline{z}-\underline{x}^{+}\right)
\end{aligned}
$$

where $\tau>0$ is referred to as the barrier parameter and is used to guide the solution along a trajectory called the central path. Equation (24) approximates the Lagrangian of problem $(22,23)$ more and more closely as $\tau$ goes to zero [9]. Here $\underline{\mu}, \underline{\varphi}$ are the dual variables associated to the equality constraints (23.1). Adopting the modified Lagrangian function (24), the necessary and sufficient conditions for the global minimum of convex problem (24) i.e. the KKT condition [13] can be derived as:

$$
\begin{gathered}
\nabla_{\underline{x}} L=Q \underline{x}+\underline{\theta} \theta \underline{\lambda}+A^{T} \underline{\mu}+\underline{\varphi}=\underline{0} \\
\nabla_{\underline{y}} L=Y \underline{\mu}-\tau \underline{e}=\underline{0} \\
\nabla_{\underline{z}} L=Z \underline{\varphi}-\tau \underline{e}=\underline{0} \\
X \underline{\lambda}-\tau \underline{e}=\underline{0} \\
\nabla_{\underline{\mu}} L=A \underline{x}+\underline{b}^{+}+\underline{y}=\underline{0} \\
\nabla_{\underline{\varphi}} L=\underline{x}+\underline{z}-\underline{x^{+}}=\underline{0} \\
\underline{x}>\underline{0}, \underline{y}>\underline{0}, \underline{z}>\underline{0}, \underline{\lambda}>\underline{0}, \underline{\mu}>\underline{0}, \underline{\varphi}>\underline{0}
\end{gathered}
$$

where $X, Y$, and $Z$ are diagonal matrices whose diagonal elements are $\underline{x}, \underline{y}, \underline{z}$ respectively. Here $\underline{\lambda}$ is another dual variable. The quantity $\underline{x}^{T} \underline{\lambda}+\underline{y}^{T} \underline{\mu}+\underline{z}^{T} \underline{\varphi}$ is termed as duality gap [9].

Applying Newton's method to the above system of equations (25), we obtain the linear system:

$$
\left[\begin{array}{cccccc}
Q & 0 & 0 & -I_{d 1} & A^{T} & I_{d 1} \\
0 & \mathrm{M} & 0 & 0 & Y & 0 \\
0 & 0 & \Phi & 0 & 0 & Z \\
\Lambda & 0 & 0 & X & 0 & 0 \\
A & I_{d 2} & 0 & 0 & 0 & 0 \\
I_{d 1} & 0 & I_{d 1} & 0 & 0 & 0
\end{array}\right]\left\{\begin{array}{c}
\Delta \underline{x} \\
\Delta \underline{y} \\
\Delta \underline{z} \\
\Delta \underline{\lambda} \\
\Delta \underline{\mu} \\
\Delta \underline{\varphi}
\end{array}\right\}=-\left\{\begin{array}{l}
\underline{r}_{x} \\
\underline{r}_{y} \\
\underline{r} \\
\underline{r}_{\lambda} \\
\underline{r}_{\mu} \\
\underline{r}_{\phi}
\end{array}\right\}
$$

where $\Lambda, \mathrm{M}$ and $\Phi$ are diagonal matrices whose diagonal elements are $\underline{\lambda}, \underline{\mu}$ and $\underline{\varphi}$, respectively. The $I_{d}$. matrices are identity matrices with appropriate dimensions. The residuals $\underline{r}_{i}$ are defined as:

$$
\begin{aligned}
& \underline{r}_{x}=Q \underline{x}+\underline{\theta} \sigma \underline{\lambda}+A^{T} \underline{\mu}+\underline{\varphi}, \quad \underline{r}=Y \underline{\mu}-\tau \underline{e}, \quad \underline{r}=Z \underline{\varphi}-\tau \underline{e}, \\
& \underline{r}_{\lambda}=X \underline{\lambda}-\tau \underline{e}, \quad \underline{r}_{\mu}=A \underline{x}+\underline{b}^{\circ}+\underline{y}, \quad \underline{r}_{\varphi}=\underline{x}+\underline{z}-\underline{x}^{+}
\end{aligned}
$$

Equation (26) can be solved progressively as:

$$
\begin{aligned}
& \Delta \underline{x}=-H^{-1} \underline{\underline{r}}, \Delta \underline{y}=-\left(\underline{r}_{\mu}+A \underline{\underline{x}}\right), \Delta \underline{\lambda}=-X^{-1}\left(\underline{r}_{\lambda}+\Lambda \Delta \underline{x}\right), \\
& \Delta \underline{z}=-\left(\underline{r}_{q}+\Delta \underline{x}\right), \Delta \underline{\mu}=-Y^{-1}\left(\underline{r}_{y}+\mathrm{M} \Delta \underline{y}\right), \Delta \underline{\varphi}=-Z^{-1}\left(\underline{r}_{z}+\Phi \Delta \underline{z}\right) \\
& \text { where } H=Q+X^{-1} \Lambda+A^{T} Y^{-1} \mathrm{M} A+Z^{-1} \Phi
\end{aligned}
$$

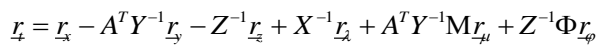

Since $Q$ is positive definite, $A$ is full row rank and during iterate $\underline{x}, \underline{y}, \underline{z}, \underline{\lambda}, \underline{\mu}, \underline{\varphi}$ remain greater than zero, $H$ is an invertible matrix.

Various algorithms can be derived depending on whether solving the primal and dual variables and on the choice of the initial point $\underline{x}^{0}$. Following [12], we can choose the values of $\underline{x}^{0}, \underline{z}^{0}, \underline{\lambda}^{0}, \underline{\mu}^{0}, \underline{\varphi}^{0}$ such that $\underline{r}_{x}=0$ and $\underline{r}_{\varphi}=0$. In our case, $\underline{x}^{0}$ may be chosen as the vector of the mean values of the upper and lower bounds or the values at the previous instant. Then, the values of $\underline{z}^{0}, \underline{\lambda}^{0}, \underline{\mu}^{0}, \underline{\varphi}^{0}$ are chosen based on the values of $\underline{x}^{0}$. Moreover, under normal situation, (23.1) can be strictly satisfied with a positive vector $\underline{y}_{0}$ and $\underline{z}^{0}$.

Based on [14], we state a feasible-initialization primal-dual path-following algorithm such as:

\section{Step 0 Initialization}

set index $k=0$, initialize the parameters $\sigma_{1}, \sigma_{2}, \sigma_{3}$ and the tolerance level $\varepsilon$, Choose the values of $\underline{x}^{0}, \underline{y}^{0}, \underline{z}^{0}, \underline{\lambda}^{0}, \underline{\mu}^{0}, \underline{\varphi}^{0}$ such that $\underline{x}^{0}>\underline{0}, \underline{y}^{0}>\underline{0}, \underline{z}^{0}>\underline{0}, \underline{\lambda}^{0}>\underline{0}, \underline{\mu}^{0}>\underline{0}, \underline{\varphi}^{0}>\underline{0}$ and the equations $\underline{r}_{x}=0, \underline{r}_{\varphi}=0$ and $\underline{r}_{\mu}=0$ are satisfied.

Step 1 Compute the duality gap,

Compute $d^{k}=\underline{k}^{k^{T}} \underline{\lambda}^{k}+\underline{y}^{k^{T}} \underline{\mu}^{k}+\underline{z}^{k^{T}} \underline{\varphi}^{k}$, if $d^{k} \leq \varepsilon$, the optimal solution is given by $\underline{\delta}^{k}=\underline{x}^{k}+\underline{\xi}^{-}$and stop, otherwise continue to step 2 .

Step 2 Update the barrier parameter $\tau^{k}$ and the search direction according to:

Compute $\quad \tau^{k}=\min \left\{\sigma_{1}, \sigma_{2} \rho^{k}\right\} \cdot \rho^{k} \quad$ where $\quad \rho^{k}=d^{k} /(2 n+m)$. Compute residuals using (27) and update search directions using (28).

Step 3 Define the step size $\alpha^{k}$ and update the variables.

Compute $\alpha^{k}$ using (29), and update the primal and dual variables using (30)

$$
\begin{gathered}
\alpha^{k}=\sigma_{3} \alpha_{\min } \\
\text { where } \quad \alpha_{\min }=\min \left(\alpha_{x}, \alpha_{y}, \alpha_{z}, \alpha_{\lambda}, \alpha_{\mu}, \alpha_{\varphi}\right) \\
\text { with } \quad\left\{\begin{array}{l}
\alpha_{\Omega}=\min _{i \text { with }(\Delta \Omega)_{i}<0}\left[-(\Omega)_{i} /(\Delta \Omega)_{i}\right], \\
\Omega \in\{\underline{x}, \underline{y}, \underline{z}, \underline{\lambda}, \underline{\mu}, \underline{\varphi}\} \\
\Delta \Omega \in\{\underline{\underline{x}}, \underline{\Delta y}, \underline{\underline{z}}, \Delta \underline{\underline{\lambda}}, \Delta \underline{\mu}, \Delta \underline{\varphi}\}
\end{array}\right.
\end{gathered}
$$


and update variables according to equations:

$$
\begin{aligned}
& \underline{x}^{k+1}=\underline{x}^{k}+\alpha^{k} \Delta \underline{x}, \underline{y}^{k+1}=\underline{y}^{k}+\alpha^{k} \Delta \underline{y}, \underline{z}^{k+1}=\underline{z}^{k}+\alpha^{k} \Delta \underline{z} \\
& \underline{\lambda}^{k+1}=\underline{\lambda}^{k}+\alpha^{k} \Delta \underline{\lambda}, \underline{\mu^{k+1}}=\underline{\mu} \underline{\mu}^{k}+\alpha^{k} \Delta \underline{\mu}, \underline{\varphi^{k+1}}=\underline{\varphi^{k}}+\alpha^{k} \Delta \underline{\varphi}
\end{aligned}
$$

Set $k=k+1$, and repeat step 1 .

\section{Numerical Simulation}

This example is taken from [15], which is concerned with the control of the unstable lateral/directional dynamics of the $\mathrm{X}-33$ vehicle at critical conditions during the entry flight. By assuming that a linear relationship exists between the rotational speed vector $\underline{v}$ and the actual actuators deflections, [15] formulates the problem as:

$$
\underline{v}=B \underline{\delta}
$$

where $\underline{v}=[p q r]^{T}, \underline{\delta}=\left[\delta_{\text {revi }}, \delta_{\text {levi }}, \delta_{r b f}, \delta_{l b f}, \delta_{r v v}, \delta_{l v r}, \delta_{\text {revo }}, \delta_{\text {levo }}\right]^{T}$ with $\delta_{\text {revi }}, \delta_{\text {levi }}=$ right and left inboard elevons; $\delta_{r b f}, \delta_{l b f}=$ right and left body flaps; $\delta_{r v r}, \delta_{l v r}=$ right and left rudders; and $\delta_{\text {revo }}$, $\delta_{\text {levo }}=$ right and left outboard elevons and $B$ is a $3 \times 8$ real valued matrix. Here we assume the rate limits for each actuator to be equal to $60 \mathrm{deg} / \mathrm{s}$.

Now to satisfy (29) as well as control surfaces limits such as $\delta_{i}^{\min } \leq \delta_{i} \leq \delta_{i}^{\max } i \in\{$ revi, levi, rbf, lbf, rvr, lvr, revo, levo $\}$, we formulate a quadratic programming problem in accordance with formation $(17,18)$ by handling $(31)$ like (13). For that, we choose the following optimality criterion at time $t+\Delta t$ :

$$
f(\underline{\delta})=\left(\underline{\delta}-\underline{\delta}_{)}\right)^{T} \Pi(\underline{\delta}-\underline{\delta})+\gamma(\underline{v}-B \underline{\delta})^{T} W_{v}(\underline{v}-B \underline{\delta})
$$

where $W_{v}$ is a diagonal matrix with diagonal positive elements $w_{p}, w_{q}, w_{r}$, $\Pi$ is a diagonal matrix with diagonal positive elements $\pi_{i} i \in I$.

\subsection{Soft Fault Scenario}

Here we present a simulation scenario where the proposed on line optimization algorithm is applied to perform a succession of complex roll maneuvers as shown in Fig 2 . There it is assumed that all actuators are fault free except for the rate limits of the left inboard elevon which changes to 20 $\mathrm{deg} / \mathrm{s}$ at $1.5 \mathrm{~s}$. The sampling time adopted by the digital control system of the different actuators is taken equal to $0.05 \mathrm{~s}$. The parameters for the interior point algorithm are chosen as $\sigma_{1}=0.1, \sigma_{2}=100, \sigma_{3}=1-10^{-6} . w_{q}=1, w_{r}=1, \pi_{i}=1$. The weights of the optimality criterion (34) are chosen as, $\gamma=$ $10^{6}, w_{p}=10, w_{q}=1, w_{r}=1, \pi_{i}=1$, where the high value of $\gamma$ is chosen such that equality constraints such as (11) are prioritized. In Fig 2 to Fig 11, the star symbol denotes the failure instant. From Fig 2, we can see that the algorithm reallocates actuators successfully to generate the desired angular rates. The time evolution of actuators position is shown in Fig 3. The number of iterations and error between desired and combined command signals are displayed in Fig 4 and Fig 5 respectively. From Fig 4, the algorithm finds the optimal solution very fast except when only a degraded objective is obtained. This degradation can be seen from Fig 5 even if the error between the generated and the desired command signals can be tolerated. From (34), it is clear that the error is introduced by the presence of the penalty function in the criterion and the error can be reduced by setting $\gamma$ larger. Fig 6 displays the speed of the failed actuator which reaches at different stages its speed limit.

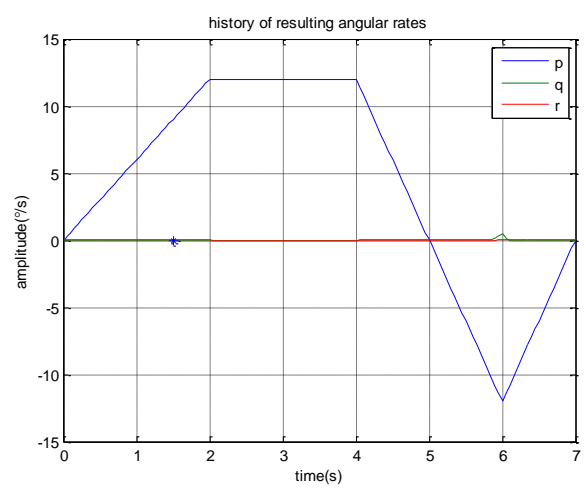

Fig 2. Time evolution of angular rates under the soft fault scenario

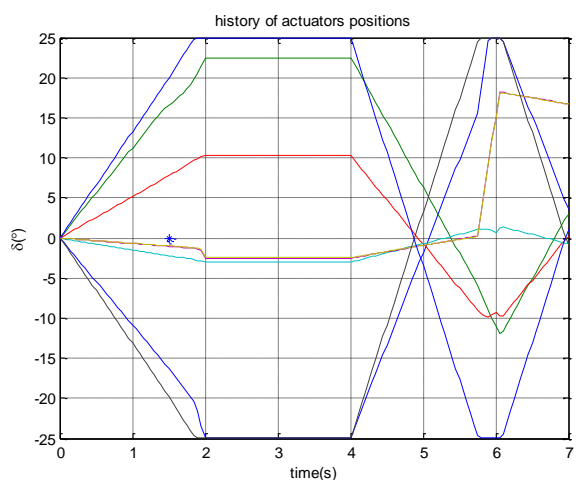

Fig 3. Time evolution of actuators positions under the soft fault scenario

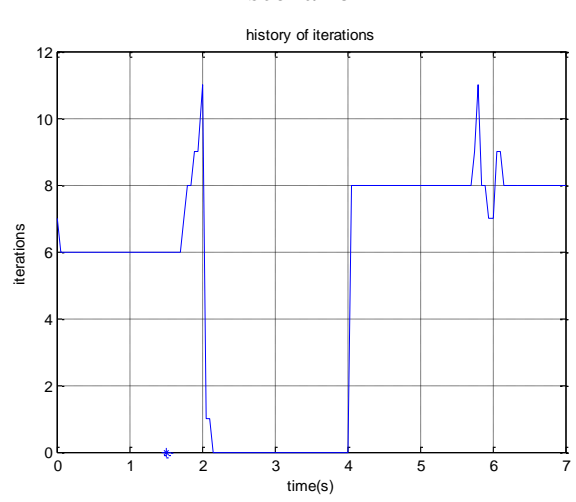

Fig 4. Number of iterations for convergence under the soft fault scenario

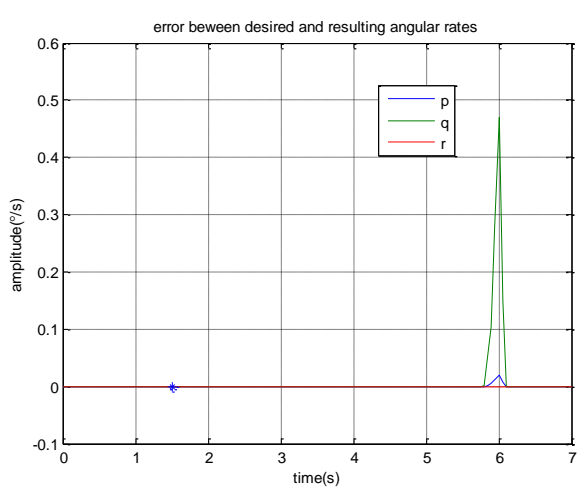

Fig 5. Angular rate errors under the soft fault scenario 


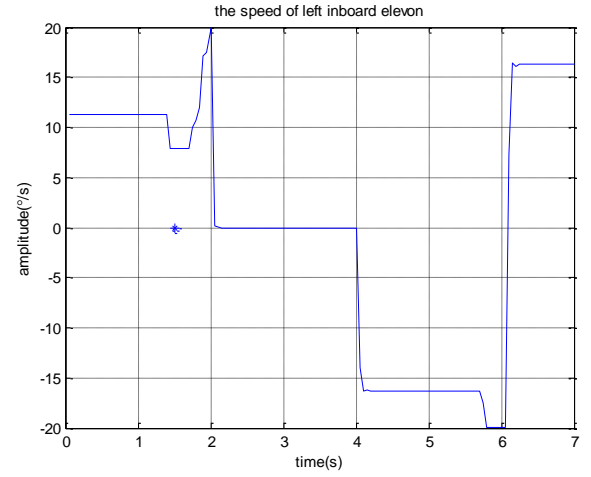

Fig 6. Example of the speed of actuator under soft fault scenario

It appears that the interior point method can handle the failure situation satisfactory even if many realistic factors such as the dynamics of the actuators and dynamic inversion controller time lags have not been considered.

\subsection{Hard Fault Scenario}

A more serious failure case occurs when an actuator remains stuck. Since a box constraint is considered by the interior point algorithm, the column corresponding to the stuck actuator must be deleted from the control effectiveness matrix and the virtual control input and limits should be changed accordingly. We simulate the case where the left inboard elevon is stuck at its previous position at $1.5 \mathrm{~s}$. Simulation parameters are the same as in section 6.1, except that the maximum amplitude of angular rate $p$ is now 10 $\mathrm{deg} / \mathrm{s}$ for the sake of realism. The corresponding results are displayed in Fig 7 to Fig 11.

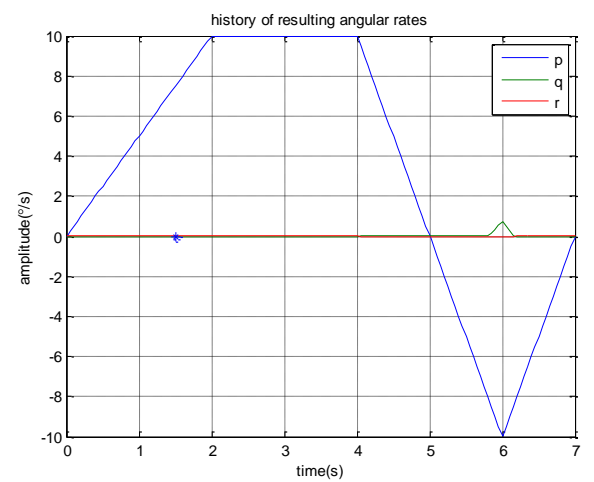

Fig 7. Time evolution of angular rates under hard fault scenario

From Fig 8 and Fig11, it can be concluded that the proposed algorithm achieves to deal effectively with a faulty actuator stuck at a fixed position. From Fig 7 and Fig 10, it appears that the resulting angular rates are sufficiently close to the desired ones. More importantly, the algorithm finds the optimal solution very fast from Fig 9 .

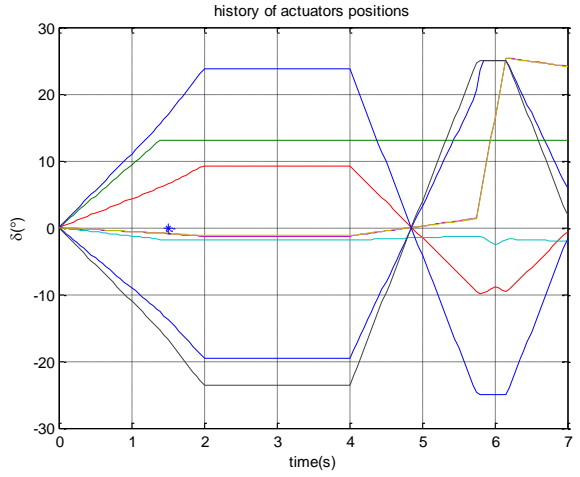

Fig 8. Evolution of actuators positions under hard fault scenario

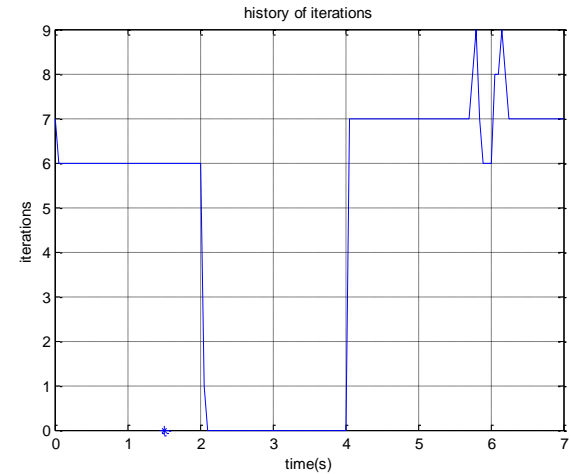

Fig 9. Number of iterations for convergence under hard fault scenario

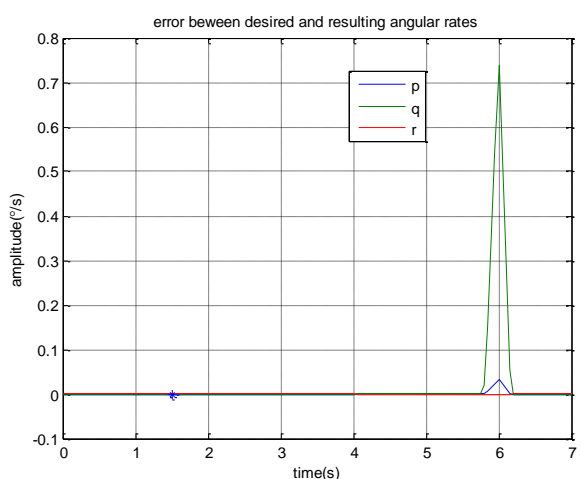

Fig 10. Error between desired and resulting angular rates under hard fault scenario

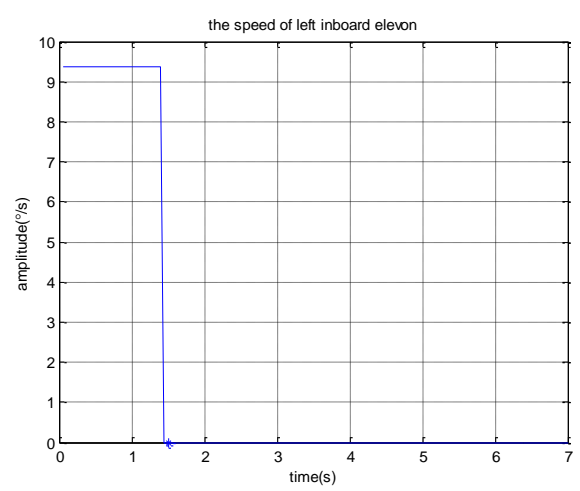

Fig 11. Example of the speed of actuator under hard fault scenario

\section{Conclusion}

In this paper, a two-stage approach is applied to manage the control surfaces of an aircraft under an actuator failure scenario. The main objective has been to maintain as much 
as possible the maneuverability of the aircraft through the fault free actuators while limiting the structural strain (maximum wing bending and flexion torques) of the aircraft. At the first stage, the necessary aerodynamics forces and torques to perform a maneuver have been synthesized by nonlinear inverse control. At the second stage, allocation of each remaining actuator to the aerodynamic forces and torques can be optimized on-line by using an interior point method which solves successive linear quadratic optimization problems. Two scenarios have been considered, one where a soft failure occurs and one where a hard failure occurs, the numerical simulation results applied to a large aircraft display the feasibility of the proposed approach.

\section{References}

[1] L. Zhong, F. Mora-Camino. A two-stage approach for managing actuators redundancy and its application to fault tolerant flight control. Chinese Journal of Aeronautics, 28(2): 469-477, 2015.

[2] M. España and G. Gilyard, Aircraft Adaptive Performance Optimization Using Redundant Control Effectors, in Automatic Control in Aerospace, 1994: 12-16.

[3] S. L. Gaulocher, C. Cumer, and D. Alazard, Aircraft load alleviation during maneuvers using optimal control surface combinations, Journal of Guidance Control and Dynamics, 30(2): 591-600, 2007

[4] J. J. Burken, P. Lu, Z. Wu, and C. Bahm, Two Reconfiguration Flight-Control Design Methods: Robust
Servomechanism and Control Allocation, Journal of Guidance, Control and Dynamics, 24(3): 482-494, 2001.

[5] L. Zhong, F. Mora-Camino, Neural Networks Based Aircraft Fault Tolerant Control, 14th AIAA/ISSMO Conference, Indiana, USA, 2012.

[6] B. Etkin, L. D. Reid, Dynamics of flight: stability and control, 3 edition, Wiley, 1995.

[7] Roux, E. , Modèle de Masse Voilure: Avions de transport civil, Ph.D. Dissertation, SupAéro-ONERA, Toulouse, France, 2006.

[8] Andrei,G., Contribution à la commande tolérante aux pannes pour la conduite du vol, Ph.D. Dissertation, INSA, Toulouse, France, 2010.

[9] D. G. Luenberger, and Y. Ye, Linear and Nonlinear Programming. Springer, 2008.

[10] J. Nocedal, S. J. Wright, Numerical Optimization, Springer, 1999.

[11] M. S. K. Lau, S. P.Yue, K. V. Ling, and J. M. Maciejowski, A Comparison of Interior Point and Active Set Methods for FPGA Implementation of Model Predictive Control, in European Control Conference, 2009: 156-161.

[12] J. A. M. Petersen, M. Bodson, Constrained quadratic programming techniques for control allocation. IEEE Trans. on Control Systems Technology, 14(1): 91-98, 2006.

[13] H. W. Kuhn, A. W. Tucker, Nonlinear programming, in Proceedings of 2nd Berkeley Symposium, Berkeley, 1951: 481-492.

[14] A. Antonious, W. Lu, Practical Optimization Algorithms and Engineering Applications. Springer, 2007

[15] M. Demenkov, Geometric Algorithms for Input Constrained Systems with Application to Flight Control, Ph.D. Dissertation, De Montfort University, UK, 2007. 